\title{
ON A CONJECTURE OF J.S. FRAME
}

\author{
NOBORU ITO*
}

\section{To Professor Kiyoshi Noshiro on His Sixtieth Birthday}

Let $\&$ be a transitive group of degree $n$, and let $\mathscr{S}_{1}$ be the stabilizer of a symbol in (s). Then we owe to J.S. Frame the following remarkable relations between the lengths $n_{i}$ of the orbits of $\mathscr{S}_{1}$ and the degrees $f_{i}$ of the absolutely irreducible components of the permutation matrix representation $\mathfrak{S S}^{*}$ of \&s:

(A) If the irreducible constituents of $\&^{*}$ are all different, then the rational number

$$
F=n^{k-2} \prod_{i=1}^{k} n_{i} / f_{i}
$$

is an integer, where $k$ is the number of the orbits of $\mathfrak{S}_{1}$.

(C) If the irreducible constituents of $\mathbb{S}^{*}$ all have rational characters, then $F$ is a square.

Further J.S. Frame made the following conjecture ([1]):

(B) If the $k$ numbers $n_{i}$ are all different, then $F$ is a square.

(B) is true for $k \leqq 3([3], \S 30)$.

Now the purpose of this short note is to show that $(B)$ is not true in general for $k=4$.

Let $L F_{r}(q)$ be the $r$-dimensional projective special linear group over the field of $q$ elements such that $p=\frac{q^{r}-1}{q-1}$ is a prime and $r$ is odd. Let $V_{r}(q)$ and $W_{r}(q)$ be the $r$-dimensional spaces of column and row vectors over the field of $q$ elements, respectively. Let $V$ and $W$ be the set of one-dimensional subspaces of $V_{r}(q)$ and $W_{r}(q)$, respectively. $\left\langle x_{1}\right\rangle \in V$ and $\left\langle y_{1}, \ldots \ldots, y_{r}\right\rangle \in W$ de$\left\langle\begin{array}{c}x_{1} \\ \vdots \\ x_{r}\end{array}\right\rangle$

note the one-dimensional subspaces generated by $\left(\begin{array}{c}x_{1} \\ \vdots \\ x_{r}\end{array}\right) \in V_{r}(q)$ and $\left(y_{1}, \ldots \ldots, y_{r}\right)$

* This research was partially supported by National Science Foundation Grand GP-5276 
$\in W_{r}(q)$, respectively.

$L F_{r}(q)$ can be considered in a natural manner as permutation groups on $V$ and also on $W$. The number of elements in $V$ and $W$ are equal to $p=\left(q^{r}-1\right) /(q-1)$, and two conjugacy classes of subgroups of $L F_{r}(q)$ of index $p$ correspond to the stabilizers of the symbols of $V$ and $W$ respectively. Let $\mathfrak{A}$ and $\mathfrak{B}$ be the stabilizers of $\left.\mid \begin{array}{c}0 \\ \vdots \\ 0 \\ 1\end{array}\right)$ and $\left.<1,0, \ldots \ldots, 0\right\rangle$ in $L F_{r}(q)$ respectively. Let $\mathfrak{P}$

be a Sylow $p$-subgroup of $L F_{r}(q)$ and let $P$ be a generating element of $\mathfrak{P}$. Then $P^{-i}\left|\begin{array}{c}0 \\ \vdots \\ 0 \\ 1\end{array}\right|(i=0, \ldots \ldots, p-1)$ and $<1,0, \ldots \ldots, 0>P^{j}(j=0, \ldots \ldots, p-1)$ are all different. Making $P^{-i}\left(\begin{array}{c}0 \\ \vdots \\ 0 \\ 1\end{array} \mid\right.$ and $<1,0, \ldots \ldots, 0>P^{j}$ correspond to $\mathfrak{A} P^{i}$ and $P^{-j} \mathfrak{B}$, respectively, let us denote the permutation representations of $L F_{r}(q)$ over $V$ and $W$ by $\boldsymbol{A}$ and $\boldsymbol{B}$ respectively.

$\mathfrak{A}$ consists of the matrices of the form $\left(\begin{array}{c}0 \\ * \\ \vdots \\ 0 \\ \rho\end{array}\right), \rho \in G F(q)$, whose determinants are equal to $1 . \quad \boldsymbol{B}(\mathfrak{X})$ has two orbits, namely, $D=\{<\ldots \ldots, 0>\}$ and $W-D$, whose lengths are equal to $k=\frac{q^{r-1}-1}{q-1}$ and $p-k$, respectively. Thus the conjugacy class of $B$ in $G$ is divided into two $A$-classes, each of which contain $k$ and $p-k$ subgroups, respectively.

$L F_{r}(q)$ admits an involutory automorphism $\tau$ such that $X^{\tau}=\left(X^{t}\right)^{-1}$ for every element $X$ of $L F_{r}(q)$, where $t$ denotes the transpose operation.

It is easy to see that $\mathfrak{A}^{\tau}$ is conjugate to $\mathfrak{B}$. In fact, $\mathfrak{A}^{\tau}$ is the stabilizer of $<0, \ldots \ldots, 0,1>$ in $L F_{r}(q)$. Let \&s be the split extension of $L F_{r}(q)$ by $\tau$. We notice that $A \tau X=A X^{\tau} \tau$ for every element $X$ of $L F_{r}(q)$.

Let us consider the following permutation representation of $\&$ by the subgroup $\mathfrak{A}$ :

$$
X \rightarrow\left(\begin{array}{ll}
\mathfrak{U}, & \mathfrak{A} P, \quad \ldots \ldots, \mathfrak{A} \tau, \quad \mathfrak{A} \tau P, \quad \ldots \ldots . \\
\mathfrak{U} X, & \mathfrak{A} P X, \ldots \ldots, \mathfrak{A} \tau X, \mathfrak{A} \tau P X, \ldots \ldots . .
\end{array}\right)
$$

Then it is easy to see that the lengths of the orbits of $\mathfrak{A}$ are equal to $1, p-1$, $k$ and $p-k$. Thus the permutation representation decomposes into four absolutely irreducible components. Then it is quite easy to see that their 
degrees are equal to $1,1, p-1$ and $p-1$. Now $F=(2 p)^{4}(p-1) k(p-k) /(p-1)^{2}$ $=(2 p)^{4} q^{r-2}$ is not a square, since $q$ is a non-square and $r$ is odd.

\section{REFERENCES}

[1] J.S. Frame, The degrees of the irreducible components of simply transitive permutation groups, Duke Math. J. 3, 8-17 (1937).

[2] J.S. Frame, The double cosets of a finite group, Bull. Amer. Math. Soc. 47, 458-467 (1941).

[3] H. Wielandt, Finite Permutation Groups, Academic Press (1964).

Nagoya University and University of Illinois Chicago, Illinois 